\title{
Interaction of Hawking radiation and a static electric charge
}

\author{
Luís C. B. Crispino \\ Instituto de Física Teórica, Universidade Estadual Paulista, Rua Pamplona 145, 01405-900, São Paulo, São Paulo, Brazil \\ and Departamento de Física, Universidade Federal do Pará, Campus Universitário do Guamá, 66075-900, Belém, Pará, Brazil \\ Atsushi Higuchi \\ Department of Mathematics, University of York, Heslington, York YO1 5DD, United Kingdom \\ George E. A. Matsas \\ Instituto de Física Teórica, Universidade Estadual Paulista, Rua Pamplona 145, 01405-900, São Paulo, São Paulo, Brazil
}

(Received 22 April 1998; published 22 September 1998)

\begin{abstract}
We investigate whether the equality found for the response of static scalar sources interacting (i) with Hawking radiation in Schwarzschild spacetime and (ii) with the Fulling-Davies-Unruh thermal bath in the Rindler wedge is maintained in the case of electric charges. We find a finite result in the Schwarzschild case, which is computed exactly, in contrast with the divergent result associated with the infrared catastrophe in the Rindler case, i.e., in the case of uniformly accelerated charges in Minkowski spacetime. Thus the equality found for scalar sources does not hold for electric charges. [S0556-2821(98)00620-1]

PACS number(s): 04.70.Dy, 04.62.+v, 41.20.Cv
\end{abstract}

It is well known that the equivalence principle played a crucial role in the development of general relativity. It continues to be tested with great success [1]. Recently many authors have asked the question whether or not a quantum version of the equivalence principle could be formulated (see, e.g., Ref. [2]). The main problem in accomplishing this task stems from the fact that states in quantum mechanics are defined globally while the equivalence principle involves only local quantities. Hence, only those phenomena which are characterized by frequencies much higher than the spacetime curvature are expected to show some equivalence for flat and curved spacetimes. (For example, Hawking radiation can be derived by requiring that physics near the black hole horizon be the same as that in Minkowski spacetime in the infinite frequency limit.) Thus, there is no a priori reason to expect any equivalence for low-frequency quantum phenomena in flat and curved spacetimes. Very recently, however, an interesting equality was found for the response of scalar sources [3]. Namely, the response rate of a static point source $q$ in Schwarzschild spacetime (with the Unruh vacuum) is equal to the response rate of the same static source in Rindler spacetime (with the Minkowski vacuum), which is

$$
R^{S}=\frac{q^{2} a}{4 \pi^{2}}
$$

provided that both sources have the same proper acceleration $a$. (We recall that a static source in Rindler spacetime is nothing but a uniformly accelerated source in Minkowski spacetime.) The response of structureless static sources is entirely due to the emission and absorption of "zerofrequency particles." Thus, this equality clearly involves low frequencies.

The choice of the quantum vacuum is crucial for this equality [4]. For instance, it would not be valid if we replaced the Unruh vacuum by the Hartle-Hawking vacuum. However, since the Unruh vacuum is more physical in the sense that it corresponds to the quantum state for a black hole formed by gravitational collapse, this equality might be pointing to some underlying equivalence principle. Thus, it is interesting to see whether or not this equality holds for other fields.

In this paper we investigate the response of a static electric charge in Schwarzschild spacetime interacting with photons of Hawking radiation (with the Unruh vacuum). We find that the response rate is finite. This immediately implies that there is no equality analogous to that found in the scalar case because the corresponding rate in Rindler spacetime is infrared divergent. We present the exact response rate for the electric charge instead of merely showing that it is infrared finite since it is a physically meaningful quantity which one could measure in principle. We first proceed to the quantization of the Maxwell field in the exterior region of Schwarzschild spacetime in some detail. Then we present the response rate and discuss it. We use natural units $c=\hbar=G=k_{B}=1$ and signature $(+---)$ throughout this paper.

The line element for the exterior region of Schwarzschild spacetime $(r>2 M)$ is given by

$$
d s^{2}=f d t^{2}-f^{-1} d r^{2}-r^{2} d \theta^{2}-r^{2} \sin ^{2} \theta d \phi^{2},
$$

where $f(r)=1-2 M / r$. We will be interested in a static charge with the current density of the form $j^{\mu}$ $=\left(j^{t}(r, \theta, \phi), 0,0,0\right)$. However, direct use of this current density would lead to indefinite results [5]. For this reason, we start with an oscillating dipole satisfying current conservation, $\nabla_{\mu} j^{\mu}=0$ :

$$
j^{\mu}=\left(j^{t}, j^{r}, 0,0\right),
$$

$$
j^{t}=\frac{\sqrt{2} q \cos E t}{r^{2} \sin \theta_{0}}\left[\delta\left(r-r_{0}\right)-\delta(r-L)\right] \delta\left(\theta-\theta_{0}\right) \delta\left(\phi-\phi_{0}\right),
$$

and 


$$
j^{r}=\frac{\sqrt{2} q E \sin E t}{r^{2} \sin \theta_{0}} \Theta\left(r-r_{0}\right) \Theta(-r+L) \delta\left(\theta-\theta_{0}\right) \delta\left(\phi-\phi_{0}\right) \text {. }
$$

Here, $\Theta(x)=1$ if $x>0$ and $\Theta(x)=0$ if $x<0$. At the end, we take the limit, $L \rightarrow \infty$ and $E \rightarrow 0$, to obtain a structureless static point charge at $(r, \theta, \phi)=\left(r_{0}, \theta_{0}, \phi_{0}\right)$. The normalization of the current has been chosen so that the time average of the squared charge, $\left(\int d \Sigma_{\mu} j^{\mu}\right)^{2}$, equals $q^{2}$. (A similar normalization was chosen for the scalar case.)

In order to quantize the Maxwell field, we use the standard Lagrangian density with a covariant gauge-fixing term:

$$
\mathcal{L}=-\sqrt{-} g\left[\frac{1}{4} F_{\mu \nu} F^{\mu \nu}+\frac{1}{2 \alpha}\left(\nabla^{\mu} A_{\mu}\right)^{2}\right]
$$

The corresponding equations of motion in the Feynman gauge $(\alpha=1)$ are

$$
\nabla_{\nu} \nabla^{\nu} A_{\mu}=0
$$

We write positive-frequency solutions to Eq. (3) with respect to the Killing field $\partial_{t}$ in the form

$$
A_{\mu}^{(n, \lambda, \omega, l, m)}=\zeta_{\mu}^{(n, \lambda, \omega, l, m)}(r, \theta, \phi) e^{-i \omega t}, \quad \omega>0,
$$

where we let $n=\rightarrow$ for the modes incoming from the past event horizon and $n=\leftarrow$ for those incoming from the past null infinity. The $l$ and $m$ are the angular momentum quantum numbers. The label $\lambda$ is for the four polarizations. The pure gauge modes with $\lambda=\mathrm{G}$ are the modes which can be written as $A_{\mu}^{(n, \mathrm{G}, \omega, l, m)}=\nabla_{\mu} \Phi$ for some scalar field $\Phi(x)$ and satisfy Lorenz condition, $\nabla^{\mu} A_{\mu}^{(n, \mathrm{G}, \omega, l, m)}=0$. The physical modes with $\lambda=$ I or II satisfy the Lorenz condition, and are not pure gauge. Finally the nonphysical modes with $\lambda=\mathrm{NP}$ do not satisfy the Lorenz condition. The Maxwell field operator can be expanded in terms of annihilation and creation operators associated with these modes as

$$
\hat{A}_{\mu}(x)=\sum_{n, \lambda, l, m} \int_{0}^{\infty} d \omega\left[a_{(i)} A_{\mu}^{(i)}(x)+a_{(i)}^{\dagger} A_{\mu}^{(i) *}(x)\right],
$$

where $(i)$ represents $(n, \lambda, \omega, l, m)$. We follow the GuptaBleuler procedure generalized to curved spacetime. Thus, we impose the condition $\nabla^{\mu} \hat{A}_{\mu}^{(+)}|p h y s\rangle=0$, where $\hat{A}_{\mu}^{(+)}$is the positive-frequency part of the operator $\hat{A}_{\mu}$, on the Hilbert space of the physical states.

For the sake of brevity, we will just write down the physical modes. Their derivation will be presented elsewhere. The modes we call the physical modes I can be written as

$$
\begin{aligned}
A_{\mu}^{(n, \mathrm{I}, \omega, l, m)}= & \omega^{3 / 2}\left(B_{t}^{(n, \mathrm{I}, \omega, l, m)}-\partial_{t} \Psi, B_{r}^{(n, \mathrm{I}, \omega, l, m)}\right. \\
& \left.-\partial_{r} \Psi,-\partial_{\theta} \Psi,-\partial_{\phi} \Psi\right)
\end{aligned}
$$

where

$$
\begin{aligned}
B_{t}^{(n, \mathrm{I}, \omega, l, m)}= & \frac{i}{M \omega}\left[(z-1) \frac{d q_{\omega l}^{n}(z)}{d z}\right. \\
& \left.+\frac{(z-1)}{(z+1)} q_{\omega l}^{n}(z)\right] Y_{l m}(\theta, \phi) e^{-i \omega t}, \\
B_{r}^{(n, \mathrm{I}, \omega, l, m)}= & \frac{(z+1)^{2}}{(z-1)} q_{\omega l}^{n}(z) Y_{l m}(\theta, \phi) e^{-i \omega t},
\end{aligned}
$$

and

$$
\square_{s} \Psi=-\frac{2 f}{r} B_{r}^{(n, \mathrm{I}, \omega, l, m)} .
$$

Here, $z \equiv r / M-1$, the $Y_{l m}(\theta, \phi)$ are the usual spherical harmonics and $l \geqslant 1$. The operator $\square_{s}$ is the Laplace-Beltrami operator for the scalar field. The $q_{\omega l}^{n}(z)$ are solutions of the differential equation

$$
\begin{gathered}
\frac{d}{d z}\left[\left(1-z^{2}\right) \frac{d q_{\omega l}^{n}}{d z}\right]+\left[l(l+1)-\frac{2}{z+1}\right. \\
\left.-M^{2} \omega^{2} \frac{(z+1)^{3}}{(z-1)}\right] q_{\omega l}^{n}=0 .
\end{gathered}
$$

The $\overrightarrow{q_{\omega l}}(z)$ satisfy the boundary codition $q_{\omega l}(z) \sim e^{i M \omega z} / z$ as $z \rightarrow \infty$. On the other hand, the $q_{\omega l}^{\leftarrow}(z)$ satisfy $q_{\omega l}^{\leftarrow}(z) \sim(z$ $-1)^{-2 i M \omega}$ as $z \rightarrow 1$. The $l=0$ solutions here can be shown to be pure gauge.

The other physical modes, which we call the physical modes II, can be written in the form

$$
A_{\mu}^{(n, \mathrm{II}, \omega, l, m)}=\left(0,0, A_{\theta}^{(n, \mathrm{II}, \omega, l, m)}, A_{\phi}^{(n, \mathrm{II}, \omega, l, m)}\right),
$$

where $l \geqslant 1$ and $A_{j}^{(n, \mathrm{II}, \omega, l, m)} \propto(z+1) q_{\omega l}^{n}(z) Y_{j}^{(l m)}(\theta, \phi) e^{-i \omega t}$, $j=\theta, \phi$. The $Y_{j}^{(l m)}(\theta, \phi)$ are the divergence-free vector spherical harmonics (see, e.g., Ref. [6]).

The normalization factors for the functions $q_{\omega l}^{n}(z)$ are determined from the canonical commutation relations of the fields by requiring suitable commutation relations for the annihilation and creation operators. It is convenient in this context to define the generalized Klein-Gordon inner product,

$$
\left(A^{(i)}, A^{(j)}\right) \equiv \int_{\Sigma} d \Sigma_{\mu} W^{\mu}\left[A^{(i)}, A^{(j)}\right],
$$

between any two modes $A_{\mu}^{(i)}$ and $A_{\mu}^{(j)}$, where the integration is performed on some Cauchy surface $\Sigma$. Here,

$$
W^{\mu}\left[A^{(i)}, A^{(j)}\right] \equiv \frac{i}{\sqrt{-g}}\left(A_{\nu}^{(i) *} \pi^{(j) \mu \nu}-A_{\nu}^{(j)} \pi^{(i) \mu \nu *}\right),
$$

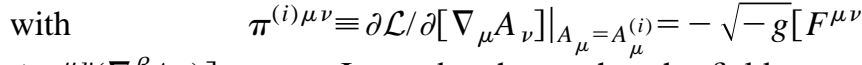

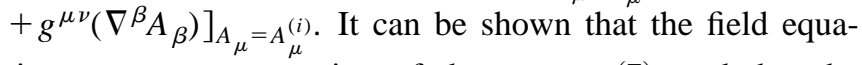
tions ensure conservation of the current (7), and that the inner product (6) is independent of the choice of the Cauchy surface $\Sigma$ as a consequence (see, e.g., Ref. [7]). Moreover, the inner product (6) is gauge invariant for physical (and 
pure gauge) modes. As has been pointed out elsewhere [8], the canonical commutation relations among fields and their conjugate momenta lead to those of the annihilation and creation operators given schematically as $\left[a_{(i)}, a_{(j)}\right]$ $=\left[a_{(i)}^{\dagger}, a_{(j)}^{\dagger}\right]=0$ and $\left[a_{(i)}, a_{(j)}^{\dagger}\right]=\left(M^{-1}\right)_{(i)(j)}$, where $M^{(i)(j)}$ $\equiv\left(A^{(i)}, A^{(j)}\right)$. The pure gauge and nonphysical modes can be chosen to be orthogonal to the physical modes with respect to the inner product (6). Thus, by requiring the usual commutation relations for annihilation and creation operators,

$$
\left[a_{(n, \lambda, \omega, l, m)}, a_{\left(n^{\prime}, \lambda^{\prime}, \omega^{\prime}, l^{\prime}, m^{\prime}\right)}^{\dagger}\right]=\delta_{n n^{\prime}} \delta_{\lambda \lambda^{\prime}} \delta_{l l^{\prime}} \delta_{m m^{\prime}} \delta\left(\omega-\omega^{\prime}\right)
$$

with $\lambda, \lambda^{\prime}$ corresponding to the physical modes I and II, we are led to the following normalization condition:

$$
\left(A^{(n, \lambda, \omega, l, m)}, A^{\left(n^{\prime}, \lambda^{\prime}, \omega^{\prime}, l^{\prime}, m^{\prime}\right)}\right)=\delta^{n n^{\prime}} \delta^{\lambda \lambda^{\prime}} \delta^{l l^{\prime}} \delta^{m m^{\prime}} \delta\left(\omega-\omega^{\prime}\right) .
$$

The classical electric charge interacts with the Maxwell field via the interaction Lagrangian density

$$
\mathcal{L}_{i n t}=\sqrt{-g} j^{\mu} A_{\mu} .
$$

Recall that the thermal bath of photons come entirely from the past event horizon in the Unruh vacuum. Therefore, we need to consider only the modes with $n=\rightarrow$. Note also that only the physical modes I are excited by the current (2), because $A_{t}=A_{r}=0$ for the physical modes II, once the nonphysical modes are appropriately chosen.

The particle emission probability with fixed angular momentum for a static charge at $\left(r_{0}, \theta_{0}, \phi_{0}\right)$ immersed in the Hawking radiation with temperature $\beta^{-1}=1 /(8 \pi M)$ is

$$
\mathcal{P}_{l m}^{e m}=\lim _{L \rightarrow+\infty} \lim _{E \rightarrow 0} \int_{0}^{+\infty} d \omega\left|\mathcal{A}_{(\rightarrow, \mathrm{I}, \omega, l, m)}^{e m}\right|^{2}\left[1+\frac{1}{e^{\omega \beta}-1}\right],
$$

where

$$
\mathcal{A}_{(\rightarrow, \mathrm{I}, \omega, l, m)}^{e m}=\left\langle\rightarrow, \mathrm{I}, \omega, l, m\left|i \int d^{4} x \sqrt{-g} j^{\mu}(x) \hat{A}_{\mu}(x)\right| 0\right\rangle
$$

is the (Boulware) vacuum emission amplitude of a photon, in the lowest order of perturbation theory. Note that in the static charge limit, $L \rightarrow \infty$ and $E \rightarrow 0$, the current will interact only with zero-energy modes. Hence, we need only the functions $q_{0 l}(z)$, which are the $\omega \rightarrow 0$ limit of the solutions $q_{\omega l}(z)$ to Eq. (5). The normalization factor determined by Eq. (8) can be calculated by the procedure used in the scalar case [3]. Thus, we find

$$
q_{0 l}(z)=\frac{2 M}{\sqrt{\pi l(l+1)}}\left[Q_{l}(z)-\frac{z-1}{l(l+1)} \frac{d Q_{l}(z)}{d z}\right],
$$

where the $Q_{l}(z)$ are the Legendre functions of the second kind. [Gauge invariance of the inner product (6) allows us to use $B^{(n, \mathrm{I}, \omega, l, m)}$ in place of $A^{(n, \mathrm{I}, \omega, l, m)}$ in determining the normalization factor.] By substituting Eq. (10) in Eq. (9) and using the differential equation satisfied by $Q_{l}(z)$, we find

$$
\frac{\mathcal{P}_{l m}^{e m}}{T}=\frac{q^{2}\left(z_{0}-1\right)^{2}}{2 \pi M l(l+1) f^{1 / 2}\left(r_{0}\right)}\left[\frac{d Q_{l}\left(z_{0}\right)}{d z_{0}}\right]^{2}\left|Y_{l m}\left(\theta_{0}, \phi_{0}\right)\right|^{2},
$$

where $T=2 \pi f^{1 / 2}\left(r_{0}\right) \delta(0)$ is the total proper time and where $z_{0}=r_{0} / M-1$. One can sum over the angular momentum quantum numbers $l$ and $m$ by using the formula

$$
\sum_{l=1}^{\infty} \frac{2 l+1}{l(l+1)}\left[\frac{d Q_{l}(z)}{d z}\right]^{2}=\frac{2 Q_{1}(z)}{\left(z^{2}-1\right)^{2}},
$$

whose derivation will be given elsewhere. The resulting total emission rate is

$$
\frac{\mathcal{P}^{e m}}{T}=\frac{q^{2} a\left(r_{0}\right)}{4 \pi^{2}} Q_{1}\left(\frac{r_{0}}{M}-1\right),
$$

where $a\left(r_{0}\right)=M r_{0}^{-2} f^{-1 / 2}\left(r_{0}\right)$ is the proper acceleration of the charge. Similarly, the particle absorption rate with fixed angular momentum is

$$
\mathcal{P}_{l m}^{a b s}=\lim _{L \rightarrow+\infty} \lim _{E \rightarrow 0} \int_{0}^{+\infty} d \omega\left|\mathcal{A}_{(\rightarrow, \mathrm{I}, \omega, l, m)}^{a b s}\right|^{2} \frac{1}{e^{\omega \beta}-1},
$$

where $\left|\mathcal{A}_{(\rightarrow, \mathrm{I}, \omega, l, m)}^{a b s}\right|=\left|\mathcal{A}_{(\rightarrow, \mathrm{I}, \omega, l, m)}^{e m}\right|$ by unitarity. As a result, the total response rate of the charge is

$$
R^{V} \equiv \frac{\mathcal{P}^{e m}}{T}+\frac{\mathcal{P}^{a b s}}{T}=\frac{q^{2} a\left(r_{0}\right)}{2 \pi^{2}} Q_{1}\left(\frac{r_{0}}{M}-1\right) .
$$

By recalling that

$$
Q_{1}(z)=\frac{z}{2} \ln \frac{z+1}{z-1}-1,
$$

it is easy to see that the response rate (11) diverges as the charge approaches the horizon and vanishes like $r_{0}^{-4}$ as $r_{0} \rightarrow \infty$. Near the horizon we find $R^{V}$ $\approx\left[q^{2} a\left(r_{0}\right) / 2 \pi^{2}\right] \ln \left[4 M a\left(r_{0}\right)\right]$. One can show using the result of Ref. [5] that, for a charge with constant acceleration $a$ in Minkowski spacetime, the infrared divergence in the total response rate is given by $\left(q^{2} a / 2 \pi^{2}\right) \ln \left(\kappa^{-1} a\right)$ if one introduces an infrared cut-off $\kappa$ for the momentum transverse to the direction of acceleration. Comparison of these two formulas shows that the finite size of the black hole acts as an infrared cut-off.

We have derived the response rate of a static electric charge outside a Schwarzschild black hole interacting with Hawking radiation in the Unruh vacuum. It differs from the result obtained for a scalar source, Eq. (1), by a factor of $2 Q_{1}\left(r_{0} / M-1\right)$. In the scalar case, it was found that the response rates of static point sources in Schwarzschild spacetime (with the Unruh vacuum) and in Rindler spacetime (with the Minkowski vacuum) are equal provided that these point sources have the same proper acceleration. Obviously this equality does not hold in the vector case since the response rate of a static charge in Rindler spacetime with the 
Minkowski vacuum, which is nothing but a uniformly accelerated charge in Minkowski spacetime, is infrared divergent as we know.

To check our procedure of defining the modes in spherical polar coordinates and normalizing them through Eq. (6), we have used it to calculate the response rate of the dipole (2) immersed in a background thermal bath in Minkowski spacetime. We numerically verified that it reproduces the standard result [9]. This would also be an interesting test for the procedure used in the quantization of the Maxwell field in Schwarzschild spacetime with the gauge $A_{0}=0$ recently discussed in Ref. [10].
Finally, we note that our results are in agreement with the widely accepted conclusion in classical electrodynamics that static charges in gravitational fields do not radiate [11-13]. This is so because the zero-frequency modes which couple to the static charge considered here do not carry energy and, consequently, cannot be identified with classical radiation.

L.C. and G.M. would like to acknowledge partial financial support from CAPES through the PICDT program and Conselho Nacional de Desenvolvimento Científico e Tecnológico, respectively.
[1] C. M. Will, Phys. Rep. 113, 345 (1984); Theory and Experiment in Gravitational Physics (Cambridge University Press, Cambridge, England, 1993); T. Damour, Gravitation, Experiment and Cosmology, Les Houches Summer School on Gravitation and Quantizations, Session 57, Les Houches, France, 1992, edited by J. Zinn-Justin and B. Julia (North Holland, Amsterdam, 1995).

[2] P. Candelas and D. W. Sciama, Phys. Rev. D 27, 1715 (1983); H. Kleinert, Quantum Equivalence Principle, Proceedings of NATO Advanced Study Institute on Functional Integration: Basics and Applications, Cargèse, France, 1996, edited by Cecile DeWitt-Morette, Pierre Cartier, and Antoine Folacci (Plenum, New York, 1997) (NATO ASI, Series B, Physics, Vol. 361).

[3] A. Higuchi, G. E. A. Matsas, and D. Sudarsky, Phys. Rev. D
56, R6071 (1997).

[4] A. Higuchi, G. E. A. Matsas, and D. Sudarsky, "Interaction of Hawking radiation with static sources outside a Schwarzschild black hole"' [Phys. Rev. D (to be published)].

[5] A. Higuchi, G. E. A. Matsas, and D. Sudarsky, Phys. Rev. D 45, R3308 (1992); 46, 3450 (1992).

[6] A. Higuchi, Class. Quantum Grav. 4, 721 (1987).

[7] J. L. Friedman, Commun. Math. Phys. 62, 247 (1978).

[8] A. Higuchi, Nucl. Phys. B325, 745 (1989).

[9] C. Itzykson and J.-B. Zuber, Quantum Field Theory (McGrawHill, New York, 1980).

[10] G. Cognola and P. Lecca, Phys. Rev. D 57, 1108 (1998).

[11] F. Rohrlich, Ann. Phys. (N.Y.) 22, 169 (1963).

[12] D. G. Boulware, Ann. Phys. (N.Y.) 124, 169 (1980).

[13] A. K. Singal, Gen. Relativ. Gravit. 27, 953 (1995). 